Open Access

\title{
The impact of aggregating serogroups in dynamic models of Neisseria meningitidis transmission
}

\author{
Keith D Poore ${ }^{1}$ and Chris T Bauch ${ }^{1,2^{*}}$
}

\begin{abstract}
Background: Neisseria meningitidis $(\mathrm{Nm})$ is a pathogen of multiple serogroups that is highly prevalent in many populations. Serogroups associated with invasive meningococcal disease (IMD) in Canada, for example, include A, $B$, $\mathrm{C}, \mathrm{W}-135, \mathrm{X}$ and $\mathrm{Y}$. IMD is a rare but serious outcome of $\mathrm{Nm}$ infection, and can be prevented with vaccines that target certain serogroups. This has stimulated the development of dynamic models to evaluate vaccine impact. However, these models typically aggregate the various $\mathrm{Nm}$ serogroups into a small number of combined groups, instead of modelling each serogroup individually. The impact of aggregation on dynamic $\mathrm{Nm}$ model predictions is poorly understood. Our objective was to explore the impact of aggregation on dynamic model predictions.

Methods: We developed two age-structured agent-based models-a 2-strain model and a 4-strain model-to simulate vaccination programs in the Canadian setting. The 2 -strain model was used to explore two different groupings: $C$, versus all other serogroups combined; and B, versus all other serogroups combined. The 4-strain model used the four groupings: C, B, Neisseria lactamica, versus all other serogroups combined. We compared the predicted impact of monovalent $C$ vaccine, quadrivalent ACWY vaccine (MCV-4), and monovalent B vaccine (4CMenB) on the prevalence of serogroup carriage under these different models.

Results: The 2-strain and 4-strain models predicted similar overall impacts of vaccines on carriage prevalence, especially with respect to the vaccine-targeted serogroups. However, there were some significant quantitative and qualitative differences. Declines in vaccine-targeted serogroups were more rapid in the 2-strain model than the 4-strain model, for both the $C$ and the $4 C M e n B$ vaccines. Sustained oscillations, and evidence for multiple attractors (i.e., different types of dynamics for the same model parameters but different initial conditions), occurred in the 4-strain model but not the 2-strain model. Strain replacement was also more pronounced in the 4-strain model, on account of the 4-strain model spreading prevalence more thinly across groups and thus enhancing competitive interactions.
\end{abstract}

Conclusions: Simplifying assumptions like aggregation of serogroups can have significant impacts on dynamic model predictions. Modellers should carefully weigh the advantages and disadvantages of aggregation when formulating models for multi-strain pathogens.

\footnotetext{
* Correspondence: cbauch@uwaterloo.ca

'Department of Mathematics and Statistics, University of Guelph, 50 Stone Road East, Guelph, ON, Canada

${ }^{2}$ Department of Applied Mathematics, University of Waterloo, 200 University

Avenue West, Waterloo, ON, Canada
} 


\section{Background}

Neisseria meningitidis $(\mathrm{Nm})$ is a significant concern to public health. Prevalence of carriage varies between 5 and $10 \%$ of the population [1]. Six main serogroups impact public health: A, B, C, W-135, X, and Y [1]. In Canada, serogroup $\mathrm{B}$ is most prevalent, although $\mathrm{C}, \mathrm{X}$, and $\mathrm{Y}$ are part of the ecological landscape as well, and are targeted by immunization programs at the time of writing [2-4]. Incidence of invasive meningococcal disease (IMD) in Canada ranges from 0.4 to 0.7 per 100,000 individuals [2], which is small compared to the disease burden in some countries, such as the "meningitis belt" of sub-Saharan Africa.

$\mathrm{Nm}$ infection is usually commensal: the pathogen typically colonizes the mucosal layer of the nasopharynx asymptomatically. $\mathrm{Nm}$ can be spread through the aerosol route, including coughing and sneezing, but also through close contact [2]. Therefore, closed environments, such as schools, households, and workplaces yield conditions conducive to transmission [5-10]. IMD incidence tends to be highest in children under 2 years of age, while $\mathrm{Nm}$ carriage tends to be highest in adolescent teenagers and young adults (17-21 years of age).

IMD symptoms develop when the colony penetrates the mucosal layer into the bloodstream, leading eventually to meningitis septicaemia [11]. Targeting the meninges and causing IMD, $N$. meningitidis can cause permanent neurological damage or fatality if not treated promptly. Many who survive IMD suffer loss of limbs or hearing. Determinants of individual susceptibility to IMD are not well understood.

There are several routes to natural immunity to Neisseria meningitis. After being infected with any serogroup of $\mathrm{Nm}$, many individuals produce anti-bodies that prevent invasion by all serogroups of the pathogen, reducing the risk of becoming infected, although the duration of immunity is unknown, and probably relatively short [12]. Another route to transient $\mathrm{Nm}$ immunity is contracting Neisseria lactamica ( $N l)$, a commensal pathogen. Though it is known that $\mathrm{Nl}$ colonizes the same loci as $\mathrm{N}$. meningitidis, the mechanism and nature of the immunity is under study and currently not well known, though ideas of creating a vaccine using $N l$ have been proposed $[13,14]$. N. lactamica colonization induces Immunoglobin $\mathrm{A}$ and Immunoglobin $\mathrm{G}$ antibody production, preventing recolonization for at least 24 weeks [15]. Though the mechanism is not clear, it is known that an immunological response to $\mathrm{Nl}$ causes an immunological response to $\mathrm{Nm}$.

$N$. meningitidis and $N$. lactamica compete with one another for the same loci in the nasopharynx, as do the various $\mathrm{N}$. meningitidis serogroups. If one pathogen is introduced to a host while the other has already colonized, the invading pathogen will not easily colonize due to competition for nutrients or adhesion [13].
Currently, vaccines reduce carriage and IMD rates in vaccinated individuals, and also unvaccinated individuals through herd immunity [16]. Previous polysaccharide vaccines were relatively ineffective, and could cause side effects [17]. However, in 2001 a conjugate vaccine that targets serogroup $\mathrm{C}$ was recommended for use in Canada for infants and children [18]. IMD incidence attributable to serogroup $\mathrm{C}$ was greatly reduced amongst the age groups that were vaccinated, as well as those that were not vaccinated [18]. However, with the reduced incidence of IMD attributable to serogroup $C$ (and presumably, carriage as well), a small gap has been left in the ecological niche: there is less asymptomatic carriage of serogroup $C$ in the nasopharyx, which, in principle, means more opportunities for other serogroups to colonize. This has caused some concern over potential vaccine-induced strain replacement [19]. We speculate that this 'ecological' strain replacement should be even stronger for vaccines against very common serogroups, such as serogroup B, if serogroups compete for space in the nasopharynx.

In 2007, Canada's National Advisory Committee on Immunization recommended a quadrivalent conjugate $\mathrm{Nm}$ vaccine, meningococcal conjugate vaccine (MCV-4), that immunizes against serogroups A, C, W-135, and Y. A routine vaccine program was recommended for adolescent individuals in areas that local epidemiology warranted the prevention of the outbreaks, while high risk individuals were also recommended for vaccination [20].

Serogroup B causes significant IMD in many countries, since it dominates the ecological landscape of its environment [21]. Vaccines against serogroup B have been difficult to produce because the capsular polysaccharides for serogroup B are too similar to human neural antigens $[22,23]$. Previous attempts required up to 20 different types of the antigen, PorA [24]. Though there have been difficulties, a vaccine has been developed using only four different components, 4CMenB [25]. With the use of $4 \mathrm{CMenB}$ in addition to MCV-4, it has been predicted that more than three quarters of the various $\mathrm{Nm}$ serogroups in Europe can be covered [26]. It is also predicted that $4 \mathrm{CMenB}$ can be used to target certain strains of serogroup $\mathrm{X}$, which no vaccine is currently able to cover [27]. 4CMenB is one step closer to a universal vaccine for $\mathrm{Nm}$, since the components found in the vaccine can also be found in all other capsule groups, thus $4 \mathrm{CMenB}$ may have an impact on other serogroups, although perhaps less efficaciously than the MCV-4 vaccine.

Previous models describing the impact of immunization on population health have aggregated all $\mathrm{Nm}$ serogroups into only two or three groups [28-31]. Some models aggregated all serogroups of $\mathrm{Nm}$ while modeling the effects of $\mathrm{Nl}$ [28]. Other models have analyzed a single vaccine applied to different age categories [2, 30,31], while some 
models compare vaccine types [2]. These models can produce valuable insights, and aggregating serogroups is a necessary model simplification. However, aggregating serogroups can also influence model projections because the ecological effects of serogroup structure and serogroup interactions cannot be fully accounted for $[32,33]$, and it is known that multi-strain models can produce qualitatively different dynamics from singlestrain models [34-38]. To our knowledge no research has directly compared the results of few-strain versus many-strain dynamic $\mathrm{Nm}$ models.

Here we develop, analyse and compare two agestructured, agent-based transmission models. The first model aggregates all serogroups into one of two categories while the second model aggregates all serogroups into one of four categories. Our objective is to compare the predicted impacts of monovalent $\mathrm{C}$, monovalent $\mathrm{B}$, and quadrivalent $\mathrm{CAWY}$ vaccines on $\mathrm{Nm}$ carriage using the two models, in order to learn more about potential biases introduced by using few-strain models to model multi-strain infectious diseases such as $\mathrm{Nm}$.

\section{Methods}

\section{4-strain model}

Our 4-strain model utilizes four groupings: (1) serogroup B, (2) serogroup C, (3) serogroups A, W-135, X, Y, 29e, and 'ungroupable' (UG) serogroups, and (4) Neisseria lactamica. We include Neisseria lactamica since it competes for the same loci on the nasopharynx as Neisseria meningitidis serogroups [13]. Serogroups A, W-135, X, and $\mathrm{Y}$ are rare in Canada but 29e and the ungroupable serogroups are more prevalent. Hence, in the 4-strain model, serogroup $\mathrm{B}$, serogroup $\mathrm{C}$, and $\mathrm{Nl}$ form their own compartments, whereas other serogroups are lumped into the same compartment with one or more other serogroups.

The population is divided into annual age cohorts: $<1$ year old, 1 year olds, 2 year olds, 3 year olds, ..., 99 year olds. Individual ages are updated monthly, moving the individual into the next age cohort after 12 simulated months. Individuals are removed each month according to all-cause, age-specific mortality rates [39] (Table S1 in Additional file 1). Individuals are born into the population at a constant rate, $\eta$, each month. A fine age structure enables comparing scenarios that distinguish between vaccinating at 12 months versus 24 months, for instance. However, for model calibration, broader age categories of $0-4,5-9,10-14,15-19$, and $20+$ years of age were used, because 5-year age intervals are used in most available $\mathrm{Nm}$ carriage prevalence data as well as in the available contact data [40].

Disease transmission includes a baseline transmission rate specific to each age group and strain, $\beta_{\mathrm{i}, \mathrm{k}}$, that is modified by an age specific contact matrix, $\mathrm{C}_{\mathrm{i}, \mathrm{j}}[40]$. The contact matrix includes both the physical and non- physical contact of individuals as $\mathrm{Nm}$ is transmitted through aerosol means. Newborns are susceptible to all serogroups of $\mathrm{Nm}$ and to $\mathrm{Nl}$. The probability per timestep that an individual of age $j$ is infected by serogroup $k$ is given by

$$
p\left(I_{i, k}\right)=1-\prod_{j=0}^{100}\left(1-\left(\frac{\beta_{i, k} C_{i, j} I_{j, k}}{N_{j}}\right)\right)
$$

where $I_{i, k}$,is the number of individuals of age $i$ infected with strain $k$ (carrying the strain asymptomatically), and $N_{j}$ is the number of individuals of age, $j$. Upon infection, individuals become a carrier of the $k^{\text {th }}$ serogroup.

No individual can be infected with more than one serogroup simultaneously. After the duration of carriage, $\tau_{1 k}$, has elapsed the individual becomes naturally immune to serogroup $k$ that infected them, for a short duration $\tau_{2 k}$ sampled from a gamma distribution. We assume that the average duration of carriage is the same for all ages and $\mathrm{Nm}$ serogroups. The average duration of carriage for $\mathrm{Nl}$ is also assumed to be the same for all ages, but differs from the average duration of carriage of $\mathrm{Nm}$ $[13,41]$. While natural immunity to serogroup $k$, individuals also have some partial cross-protection against other serogroups, so that the probability per timestep that an individual of age $j$ is infected by some other serogroup $k$ is given by

$$
p\left(I_{i, k}\right)=1-\prod_{i=0}^{100}\left(1-\left(\frac{\beta_{i, k} C_{i, j} I_{j, k}}{N_{j}}\right)\left(1-\sigma_{l}\right)\right)
$$

where, $0<\sigma_{1}<1$ is the cross-immunity conferred by previous infection by serogroup $l \neq k$. Cross-immunity lasts the same period of time as strain-specific immunity. Details on all parameter values appear in Additional file 1: Table S1.

\section{2-strain model}

The 2-strain model aggregates $\mathrm{Nl}$ and $\mathrm{Nm}$ serogroups, but it uses two groupings instead of four. The first version of the 2-strain model uses the groups: $\mathrm{C}$ versus $\mathrm{Nl}$ and all other $\mathrm{Nm}$ serogroups aggregated (A/B/W-135/X/ $\mathrm{Y} / 29 \mathrm{e} / \mathrm{UG} / N l$ ). The second version uses the groups $\mathrm{B}$ versus $\mathrm{Nl}$ and all other $\mathrm{Nm}$ serogroups aggregated (A/C/ $\mathrm{W}-135 / \mathrm{X} / \mathrm{Y} / 29 \mathrm{e} / \mathrm{UG} / \mathrm{Nl}$ ). The first version is used to simulate the impact of monovalent $C$ vaccine and compare it to the simulated impact of the monovalent $C$ vaccine using the 4-strain model. Likewise, the second version is used to simulate the impact of monovalent $B$ vaccine and compare it to the simulated impact using the 4-strain model. The 2-strain model is otherwise identical to the 4-strain model. In both versions, the duration of carriage and immunity was assumed to be the duration of $\mathrm{Nm}$ and was set by sampling from a gamma distribution. 


\section{Parameterization and uncertainty analysis}

We used Canadian demographic and epidemiologic data to parameterize the model. The baseline natural history and demographic parameters were taken directly from the literature, while the baseline transmission rates $\beta_{\mathrm{i}, \mathrm{k}}$ were calibrated so that the age-stratified serogroupspecific carriage prevalence in the model matched the empirical data, within specified ranges of acceptability (Figure S1 in Additional file 1 and Table S2 in Additional file 1 for the 4-strain model) [2, 3]. In particular, initial values of $\beta_{\mathrm{i}, \mathrm{k}}$ were assumed for each simulation; every 100 simulated years, the code checked whether the modelled age-specific carriage prevalence was within the range of empirical acceptability based on seroprevalence data, for all of the ranges ( 20 for the 4-strain model, 10 for the 2 -strain model); if so, those calibrated values of $\beta_{\mathrm{i}, \mathrm{k}}$ were used in the simulation, and if not, the values of $\beta_{\mathrm{i}, \mathrm{k}}$ were adjusted upward or downward as appropriate and the process was repeated for another 100 simulation years until the target ranges were attained.

The degree of cross-protection is not well known, so we used values from an in-vitro cross-reactivity study [42] (see Table S1 in Additional file 1 for baseline parameter values; see Tables S2, S3, S4 for the average $\beta_{i, k}$ values in Additional file 1). 50 realizations for each vaccine scenario were thereby produced, each through this calibration procedure. 50 realizations were used because this was found to be a sufficient number of realizations to produce representative averages of the (stochastic) agent-based model, at baseline parameter values. All results reported are the averages and standard deviations of the 50 realizations except where otherwise noted.

The calibration targets for the 4-strain model were obtained by adding the carriage prevalence of the constituent serogroups (Table S5 in Additional file 1). Similarly, the calibration of the 2-strain model, the acceptability ranges for prevalence of carriage were different for each age group and serogroup (Tables S6, S7 in Additional file 1).

In the case of the 4-strain model, a significant number of parameter sets yielded dynamics where serogroup prevalence oscillated over time, indicating the likely presence of multiple attractors. These parameter sets were not included in the baseline analysis, but are discussed separately at the end of the Results section.

\section{Vaccine scenarios and assumptions}

We explored nine vaccination scenarios with the 4strain model (Table 1) and six scenarios with the 2strain model (Table 2). Vaccination begins at $t=150$ years (which provides enough "burn-in" to discard transients) and is continued for 75 years ( $t_{\text {end }}=225$ years).

For the 4-strain model, scenarios 1-3 use a monovalent $C$ vaccine, scenarios 4-6 use a quadrivalent ACWY vaccine $(\mathrm{MCV}-4)$, and scenarios 7-9 use a monovalent
Table 1 Vaccine Scenarios for the 4-strain model

\begin{tabular}{lllll}
\hline \multicolumn{3}{l}{ 4-strain Vaccine Scenarios } & & \\
\hline & Age of Immunization & Vaccine Type & Efficacy & Coverage \\
\hline 1 & 12 months & $C$ & $97 \%$ & $90 \%$ \\
2 & 12 years & $C$ & $97 \%$ & $80 \%$ \\
3 & 12 months and 12 years & $C$ & $97 \%$ & $90 \% / 80 \%$ \\
4 & 12 months & MCV-4 & $97 \%$ & $90 \%$ \\
5 & 12 years & MCV-4 & $97 \%$ & $80 \%$ \\
6 & 12 months and 12 years & MCV-4 & $97 \%$ & $90 \% / 80 \%$ \\
7 & 12 months & $4 C M e n B$ & $64 \%$ & $90 \%$ \\
8 & 12 years & 4CMenB & $64 \%$ & $80 \%$ \\
9 & 12 months and 12 years & 4CMenB & $64 \%$ & $90 \% / 80 \%$
\end{tabular}

The efficacy of the conjugate $C$ vaccine and the MCV-4 vaccine are assumed to be $97 \%[41,46]$. The 4 CMenB vaccine efficacy is assumed to be $64 \%$ based on [44]. The duration of vaccine protection is assumed to be 4 years

$B$ vaccine (4CMenB). For the 2 -strain model, scenarios 1-3 use a monovalent $C$ vaccine and scenarios 4-6 use the $4 \mathrm{CMenB}$. Coverage of toddlers and infants was assumed to be $90 \%$ and coverage for adolescents was assumed to be $80 \%$, based on Quebec coverage rates $[38,43]$. The conjugate $\mathrm{C}$ and the $\mathrm{MCV}-4$ vaccines were assumed to be $97 \%$ efficacious in protecting inoculated individuals, while the $\mathrm{B}$ vaccine was assumed to be $64 \%$ efficacious [44].

Vaccination operates in an "all-or-none" fashion, such that individuals who are efficaciously vaccinated receive full protection from infection and cannot transmit infection to others, while individuals who are not efficaciously vaccinated receive no protection and remain susceptible. Individuals are only protected against serogroups included in the vaccine (i.e., none of the vaccines, including 4CMenB, confer cross-immunity). Individuals are randomly selected each month to be vaccinated according to the vaccine coverage for their age group, and each vaccinated person is protected with a probability equal to the vaccine efficacy.

Table 2 Vaccine scenarios for the 2-strain model

\begin{tabular}{lllll}
\hline \multicolumn{2}{l}{ 2-strain Vaccine Scenarios } & & \\
\hline 10 & Age of Immunization & Vaccine Type & Efficacy & Coverage \\
11 & 12 years & C & $97 \%$ & $90 \%$ \\
12 & 12 months and 12 years & $C$ & $97 \%$ & $80 \%$ \\
13 & 12 months & 4CMenB & $64 \%$ & $90 \%$ \\
14 & 12 years & 4CMenB & $64 \%$ & $80 \%$ \\
15 & 12 months and 12 years & 4CMenB & $64 \%$ & $90 \% / 80 \%$
\end{tabular}

The efficacy of the conjugate $C$ vaccine is $97 \%$ [46]. The 4CMenB vaccine efficacy is assumed to be $64 \%$ based on [44]. The duration of vaccine protection is assumed to be 4 years 
Table 3 Reduction in prevalence of carriage with the Conjugate C vaccine

\begin{tabular}{|c|c|c|c|c|c|c|c|}
\hline Vaccine Program & Model Type & Serogroup & $t=149$ & $t=160$ & $t=175$ & $t=190$ & Relative Change \\
\hline \multirow[t]{16}{*}{1} & 2-strain model & $C$ & 0.005 & 0.003 & 0.000 & 0.000 & -0.760 \\
\hline & & & $(0.012)$ & $(0.007)$ & $(0.000)$ & $(0.000)$ & $(0.427)$ \\
\hline & & Other & 0.109 & 0.108 & 0.107 & 0.108 & -0.009 \\
\hline & & & $(0.008)$ & $(0.008)$ & $(0.010)$ & $(0.009)$ & $(0.054)$ \\
\hline & & Total & 0.115 & 0.111 & 0.107 & 0.108 & -0.050 \\
\hline & & & $(0.020)$ & $(0.015)$ & $(0.011)$ & $(0.009)$ & $(0.068)$ \\
\hline & 4-strain model & B & 0.031 & 0.030 & 0.031 & 0.031 & 0.014 \\
\hline & & & $(0.013)$ & $(0.010)$ & $(0.012)$ & $(0.014)$ & $(0.276)$ \\
\hline & & C & 0.013 & 0.008 & 0.000 & 0.000 & -0.960 \\
\hline & & & $(0.012)$ & $(0.008)$ & $(0.001)$ & $(0.000)$ & $(0.196)$ \\
\hline & & Other & 0.045 & 0.047 & 0.050 & 0.050 & 0.357 \\
\hline & & & $(0.028)$ & $(0.026)$ & $(0.021)$ & $(0.022)$ & $(0.860)$ \\
\hline & & $N I$ & 0.025 & 0.025 & 0.025 & 0.025 & -0.011 \\
\hline & & & $(0.003)$ & $(0.002)$ & $(0.002)$ & $(0.004)$ & $(0.082)$ \\
\hline & & Total & 0.114 & 0.110 & 0.106 & 0.106 & -0.070 \\
\hline & & & $(0.056)$ & $(0.047)$ & $(0.036)$ & $(0.039)$ & $(0.102)$ \\
\hline \multirow[t]{16}{*}{2} & 2-strain model & C & 0.006 & 0.002 & 0.000 & 0.000 & -0.840 \\
\hline & & & $(0.011)$ & $(0.004)$ & $(0.000)$ & $(0.000)$ & $(0.367)$ \\
\hline & & Other & 0.108 & 0.110 & 0.108 & 0.107 & -0.007 \\
\hline & & & $(0.011)$ & $(0.009)$ & $(0.009)$ & $(0.009)$ & $(0.058)$ \\
\hline & & Total & 0.114 & 0.112 & 0.108 & 0.107 & -0.053 \\
\hline & & & $(0.022)$ & $(0.013)$ & $(0.010)$ & $(0.009)$ & $(0.073)$ \\
\hline & 4-strain model & B & 0.031 & 0.031 & 0.031 & 0.032 & 0.064 \\
\hline & & & $(0.015)$ & $(0.012)$ & $(0.013)$ & $(0.012)$ & $(0.259)$ \\
\hline & & C & 0.015 & 0.006 & 0.000 & 0.000 & -1.00 \\
\hline & & & $(0.014)$ & $(0.006)$ & $(0.001)$ & $(0.000)$ & $(0.000)$ \\
\hline & & Other & 0.047 & 0.050 & 0.051 & 0.052 & 0.412 \\
\hline & & & $(0.030)$ & $(0.027)$ & $(0.022)$ & $(0.020)$ & $(1.643)$ \\
\hline & & NI & 0.026 & 0.025 & 0.025 & 0.025 & -0.014 \\
\hline & & & $(0.003)$ & $(0.002)$ & $(0.003)$ & $(0.003)$ & $(0.078)$ \\
\hline & & Total & 0.118 & 0.112 & 0.107 & 0.108 & -0.076 \\
\hline & & & $(0.061)$ & $(0.047)$ & $(0.038)$ & $(0.035)$ & $(0.097)$ \\
\hline 3 & 2-strain model & C & 0.007 & 0.001 & 0.000 & 0.000 & -0.840 \\
\hline
\end{tabular}


Table 3 Reduction in prevalence of carriage with the Conjugate C vaccine (Continued)

\begin{tabular}{|c|c|c|c|c|c|c|}
\hline & & $(0.012)$ & $(0.002)$ & $(0.000)$ & $(0.000)$ & $(0.367)$ \\
\hline & \multirow[t]{2}{*}{ Other } & 0.109 & 0.110 & 0.108 & 0.108 & -0.007 \\
\hline & & $(0.011)$ & $(0.030)$ & $(0.010)$ & $(0.010)$ & $(0.060)$ \\
\hline & \multirow[t]{2}{*}{ Total } & 0.116 & 0.111 & 0.108 & 0.108 & -0.063 \\
\hline & & $(0.022)$ & $(0.032)$ & $(0.010)$ & $(0.010)$ & $(0.087)$ \\
\hline 4-strain model & \multirow[t]{2}{*}{ B } & 0.031 & 0.034 & 0.032 & 0.031 & 0.064 \\
\hline & & $(0.015)$ & $(0.017)$ & $(0.016)$ & $(0.013)$ & $(0.259)$ \\
\hline & \multirow[t]{2}{*}{ C } & 0.014 & 0.004 & 0.000 & 0.000 & -1.00 \\
\hline & & $(0.010)$ & $(0.007)$ & $(0.001)$ & $(0.000)$ & $(0.000)$ \\
\hline & \multirow[t]{2}{*}{ Other } & 0.046 & 0.044 & 0.048 & 0.052 & 0.412 \\
\hline & & $(0.024)$ & $(0.027)$ & $(0.022)$ & $(0.020)$ & $(-0.004)$ \\
\hline & \multirow[t]{2}{*}{ NI } & 0.025 & 0.025 & 0.025 & 0.025 & -0.014 \\
\hline & & $(0.002)$ & $(0.003)$ & $(0.002)$ & $(0.003)$ & $(0.078)$ \\
\hline & \multirow[t]{2}{*}{ Total } & 0.115 & 0.108 & 0.105 & 0.108 & -0.061 \\
\hline & & $(0.052)$ & $(0.054)$ & $(0.040)$ & $(0.036)$ & $(0.103)$ \\
\hline
\end{tabular}

Reduction in the prevalence of carriage when an immunization program with the
beginning at $\mathrm{t}=150$ years. Results for both 2 -strain and 4 -strain models are shown 


\section{Results}

The overall prevalence of carriage decreases with the introduction of vaccination, regardless of the serogroup targeted by the vaccine or the age of immunization (Tables 3, 4, 5). Herd immunity was also observed for every vaccine scenario, since prevalence declined in age groups not targeted by the vaccine. We compare the 2-strain and 4-strain model predictions for each vaccine type in the following subsections.

\section{Vaccine}

In the 4-strain model, introducing the $C$ vaccine causes the prevalence of serogroup $C$ to decline quickly (Fig. 1a-c, see also Additional file 1: Figure S3a-c for time series plots with standard deviation bars on model outputs). The 2-strain model sees a more rapid reduction in the prevalence of carriage for serogroup $C$ (Fig. 1d-f, see also Additional file 1: Figure S3d-f). In the 2-strain model, serogroup $C$ prevalence is reduced to almost zero by approximately $\mathrm{t}=165$ years, while in the 4-strain model this occurs by approximately $t=170$ years. After 15 years, the prevalence of carriage is reduced by $88.1 \%, 95.0 \%$, and $97.3 \%$ for the 4 -strain model, compared to $96.3 \%, 96.1 \%$, and $99.9 \%$ for the 2-strain model, for vaccine programs $1-3$ respectively ( $\mathrm{p}=0.0002,0.0008$, and 0.0001 , respectively) (Table 3).

The 4-strain model predicts slight strain replacement caused by the $\mathrm{C}$ vaccine: the prevalence of "Other"

Table 4 Reduction in prevalence of carriage with the MVC-4 vaccine

\begin{tabular}{|c|c|c|c|c|c|c|c|}
\hline Vaccine Program & Model Type & Serogroup & $t=149$ & $t=160$ & $t=175$ & $t=190$ & Relative Change \\
\hline \multirow[t]{10}{*}{4} & 4-strain model & $B$ & 0.031 & 0.031 & 0.035 & 0.036 & 0.202 \\
\hline & & & $(0.014)$ & $(0.013)$ & $(0.013)$ & $(0.010)$ & $(0.259)$ \\
\hline & & C & 0.015 & 0.011 & 0.001 & 0.000 & -0.958 \\
\hline & & & $(0.013)$ & $(0.009)$ & $(0.001)$ & $(0.000)$ & $(0.198)$ \\
\hline & & Other & 0.045 & 0.037 & 0.011 & 0.001 & -0.974 \\
\hline & & & $(0.026)$ & $(0.022)$ & $(0.010)$ & $(0.003)$ & $(0.052)$ \\
\hline & & $\mathrm{NI}$ & 0.025 & 0.024 & 0.023 & 0.022 & -0.118 \\
\hline & & & $(0.003)$ & $(0.002)$ & $(0.002)$ & $(0.003)$ & $(0.080)$ \\
\hline & & Total & 0.117 & 0.102 & 0.070 & 0.059 & -0.492 \\
\hline & & & $(0.056)$ & $(0.047)$ & $(0.027)$ & $(0.017)$ & $(0.060)$ \\
\hline \multirow[t]{10}{*}{5} & 4-strain model & B & 0.030 & 0.032 & 0.035 & 0.035 & 0.206 \\
\hline & & & $(0.014)$ & $(0.015)$ & $(0.016)$ & $(0.017)$ & $(0.275)$ \\
\hline & & C & 0.013 & 0.006 & 0.000 & 0.000 & -0.920 \\
\hline & & & $(0.015)$ & $(0.008)$ & $(0.002)$ & $(0.000)$ & $(0.271)$ \\
\hline & & Other & 0.046 & 0.031 & 0.008 & 0.000 & -0.971 \\
\hline & & & $(0.028)$ & $(0.020)$ & $(0.009)$ & $(0.001)$ & $(0.139)$ \\
\hline & & $\mathrm{Nl}$ & 0.026 & 0.024 & 0.023 & 0.023 & -0.106 \\
\hline & & & $(0.007)$ & $(0.003)$ & $(0.002)$ & $(0.005)$ & $(0.135)$ \\
\hline & & Total & 0.115 & 0.093 & 0.067 & 0.059 & -0.487 \\
\hline & & & $(0.064)$ & $(0.046)$ & $(0.029)$ & $(0.023)$ & $(0.087)$ \\
\hline \multirow[t]{10}{*}{6} & 4-strain model & B & 0.029 & 0.031 & 0.033 & 0.034 & 0.244 \\
\hline & & & $(0.019)$ & $(0.017)$ & $(0.019)$ & $(0.020)$ & $(0.352)$ \\
\hline & & $C$ & 0.016 & 0.017 & 0.000 & 0.000 & -0.960 \\
\hline & & & $(0.019)$ & $(0.009)$ & $(0.000)$ & $(0.000)$ & $(0.196)$ \\
\hline & & Other & 0.043 & 0.021 & 0.003 & 0.000 & -0.957 \\
\hline & & & $(0.034)$ & $(0.017)$ & $(0.005)$ & $(0.000)$ & $(0.196)$ \\
\hline & & $\mathrm{Nl}$ & 0.025 & 0.024 & 0.022 & 0.022 & -0.121 \\
\hline & & & $(0.004)$ & $(0.002)$ & $(0.003)$ & $(0.002)$ & $(0.066)$ \\
\hline & & Total & 0.113 & 0.082 & 0.059 & 0.057 & -0.499 \\
\hline & & & $(0.075)$ & $(0.045)$ & $(0.028)$ & $(0.023)$ & $(0.090)$ \\
\hline
\end{tabular}


Table 5 Reduction in prevalence of carriage with the 4 CMenB vaccine

\begin{tabular}{|c|c|c|c|c|c|c|c|}
\hline Vaccine Program & Model Type & Serogroup & $t=149$ & $t=160$ & $t=175$ & $t=190$ & Relative Change \\
\hline \multirow[t]{16}{*}{7} & \multirow[t]{6}{*}{ 2-strain model } & \multirow[t]{2}{*}{ B } & 0.027 & 0.021 & 0.008 & 0.000 & -0.996 \\
\hline & & & $(0.010)$ & $(0.008)$ & $(0.005)$ & $(0.000)$ & $(0.006)$ \\
\hline & & \multirow[t]{2}{*}{ Other } & 0.109 & 0.109 & 0.107 & 0.107 & -0.017 \\
\hline & & & $(0.009)$ & $(0.007)$ & $(0.007)$ & $(0.009)$ & $(0.056)$ \\
\hline & & \multirow[t]{2}{*}{ Total } & 0.136 & 0.131 & 0.115 & 0.107 & -0.209 \\
\hline & & & (0.019) & $(0.015)$ & $(0.012)$ & $(0.009)$ & $(0.052)$ \\
\hline & \multirow[t]{10}{*}{ 4-strain model } & \multirow[t]{2}{*}{ B } & 0.031 & 0.030 & 0.022 & 0.004 & -0.861 \\
\hline & & & $(0.014)$ & $(0.023)$ & $(0.015)$ & $(0.014)$ & $(0.000)$ \\
\hline & & \multirow[t]{2}{*}{ C } & 0.016 & 0.016 & 0.019 & 0.026 & 0.665 \\
\hline & & & $(0.014)$ & $(0.013)$ & $(0.015)$ & $(0.021)$ & $(0.007)$ \\
\hline & & \multirow[t]{2}{*}{ Other } & 0.046 & 0.039 & 0.015 & 0.028 & -0.394 \\
\hline & & & $(0.028)$ & $(0.046)$ & $(0.032)$ & $(0.054)$ & $(0.026)$ \\
\hline & & \multirow[t]{2}{*}{ NI } & 0.026 & 0.025 & 0.023 & 0.024 & -0.083 \\
\hline & & & $(0.003)$ & $(0.004)$ & $(0.003)$ & $(0.004)$ & $(0.001)$ \\
\hline & & \multirow[t]{2}{*}{ Total } & 0.119 & 0.109 & 0.079 & 0.082 & -0.308 \\
\hline & & & $(0.059)$ & $(0.086)$ & $(0.066)$ & $(0.093)$ & $(0.240)$ \\
\hline \multirow[t]{16}{*}{8} & \multirow[t]{6}{*}{ 2-strain model } & \multirow[t]{2}{*}{ B } & 0.027 & 0.015 & 0.004 & 0.000 & -0.995 \\
\hline & & & $(0.007)$ & $(0.006)$ & $(0.006)$ & $(0.000)$ & $(-0.006)$ \\
\hline & & \multirow[t]{2}{*}{ Other } & 0.110 & 0.109 & 0.107 & 0.108 & -0.016 \\
\hline & & & $(0.006)$ & $(0.010)$ & $(0.011)$ & $(0.010)$ & $(0.004)$ \\
\hline & & \multirow[t]{2}{*}{ Total } & 0.137 & 0.124 & 0.111 & 0.108 & -0.210 \\
\hline & & & $(0.012)$ & $(0.016)$ & $(0.016)$ & $(0.010)$ & $(0.048)$ \\
\hline & \multirow[t]{10}{*}{ 4-strain model } & \multirow[t]{2}{*}{ B } & 0.033 & 0.025 & 0.016 & 0.003 & -0.900 \\
\hline & & & $(0.016)$ & $(0.019)$ & $(0.018)$ & $(0.013)$ & $(0.003)$ \\
\hline & & \multirow[t]{2}{*}{ C } & 0.015 & 0.019 & 0.023 & 0.030 & 0.931 \\
\hline & & & $(0.020)$ & $(0.022)$ & $(0.024)$ & $(0.029)$ & $(0.009)$ \\
\hline & & \multirow[t]{2}{*}{ Other } & 0.044 & 0.021 & 0.014 & 0.024 & -0.444 \\
\hline & & & $(0.027)$ & $(0.032)$ & $(0.029)$ & $(0.038)$ & $(0.011)$ \\
\hline & & \multirow[t]{2}{*}{ NI } & 0.026 & 0.024 & 0.023 & 0.024 & -0.075 \\
\hline & & & $(0.002)$ & $(0.004)$ & $(0.003)$ & $(0.003)$ & $(0.001)$ \\
\hline & & \multirow[t]{2}{*}{ Total } & 0.117 & 0.089 & 0.076 & 0.081 & -0.317 \\
\hline & & & $(0.065)$ & $(0.077)$ & $(0.074)$ & $(0.083)$ & $(0.195)$ \\
\hline \multirow[t]{12}{*}{9} & 2-strain model & B & 0.027 & 0.013 & 0.001 & 0.000 & -0.027 \\
\hline & & & $(0.010)$ & $(0.007)$ & $(0.002)$ & $(0.000)$ & $(0.010)$ \\
\hline & & Other & 0.109 & 0.110 & 0.107 & 0.107 & -0.999 \\
\hline & & & $(0.010)$ & $(0.008)$ & $(0.008)$ & $(0.009)$ & $(-0.001)$ \\
\hline & & Total & 0.135 & 0.123 & 0.108 & 0.107 & -0.206 \\
\hline & & & $(0.020)$ & $(0.015)$ & $(0.011)$ & $(0.009)$ & $(0.057)$ \\
\hline & 4-strain model & B & 0.031 & 0.025 & 0.009 & 0.003 & -0.905 \\
\hline & & & $(0.019)$ & $(0.020)$ & $(0.018)$ & $(0.013)$ & $(-0.006)$ \\
\hline & & C & 0.014 & 0.016 & 0.023 & 0.024 & 0.736 \\
\hline & & & $(0.017)$ & $(0.020)$ & $(0.025)$ & $(0.023)$ & $(0.006)$ \\
\hline & & Other & 0.042 & 0.022 & 0.015 & 0.027 & -0.364 \\
\hline & & & $(0.035)$ & $(0.038)$ & $(0.039)$ & $(0.048)$ & $(0.013)$ \\
\hline
\end{tabular}


Table 5 Reduction in prevalence of carriage with the 4CMenB vaccine (Continued)

\begin{tabular}{llllll}
$N 1$ & 0.025 & 0.023 & 0.023 & 0.024 & -0.060 \\
& $(0.003)$ & $(0.003)$ & $(0.004)$ & $(0.024)$ & $(0.002)$ \\
Total & 0.112 & 0.085 & 0.070 & 0.078 & -0.302 \\
& $(0.075)$ & $(0.082)$ & $(0.085)$ & $(0.089)$ & $(0.264)$ \\
\hline
\end{tabular}

Reduction in the prevalence of carriage when an immunization program with the 4CMenB vaccine is scheduled for 12 month (program 7), 12 years (program 8), and both 12 months and 12 years (program 9) beginning at $t=150$ years. Results for both 2 -strain and 4-strain models are shown

increases slightly while the prevalence of $N l$ and $B$ remain constant in vaccine programs 2 and 3 , whereas the 2 -strain model shows very little evidence of strain replacement, on account of the significant difference in prevalence between serogroup $C$ and "Other", which includes the very common species Neisseria lactamica. This has implications for disease control since different serogroups cause differing pathogenicity.

\section{MCV-4 Vaccine}

Vaccine programs 4 to 6 introduced a quadrivalent ACWY vaccine. In the 4-strain model, this reduced serogroup C as well as the "other" serogroup which partially includes A, W-135 and Y. Similar to the monovalent $\mathrm{C}$ vaccine, it takes about 20 years to reduce the prevalence of carriage serogroup $\mathrm{C}$ to almost zero.
However, it requires an additional 10 years for the "other" serogroup to be reduced to the same level (Fig. 2a-c, see also Additional file 1: Figure S4a-c). The overall reduction in $\mathrm{Nm}$ prevalence was greater than for the $\mathrm{C}$ vaccine, on account of the MCV-4 vaccine including more serogroups. The MCV-4 vaccine causes strain replacement of serogroup B, but not of $N l$, which is least affected by competition because it has a different duration of carriage than is typical for $\mathrm{Nm}$ serogroups.

In the 4-strain model, the MCV-4 vaccine programs reduced the overall prevalence of carriage by $49.2 \%$, $48.7 \%$, and $49.9 \%$ after 40 years, compared to the reduction of $7.0 \%, 7.6 \%$, and $6.1 \%$ under the $C$ vaccine, for vaccine programs $1-3$ respectively $(\mathrm{p}=<0.0001$, $<0.0001$, and $<0.0001$, respectively) (Table 4 ). The reduction in overall prevalence of carriage is higher for the

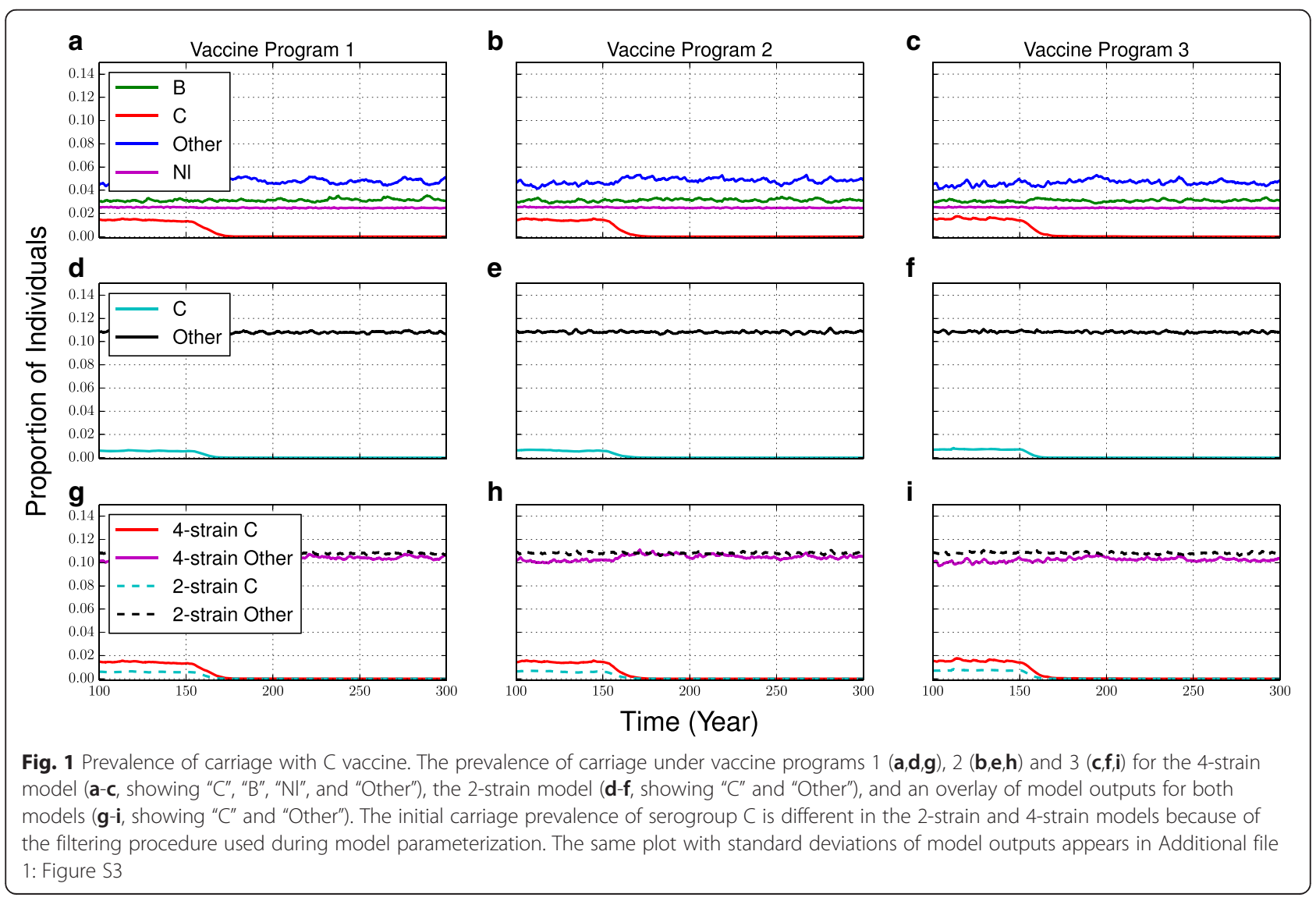




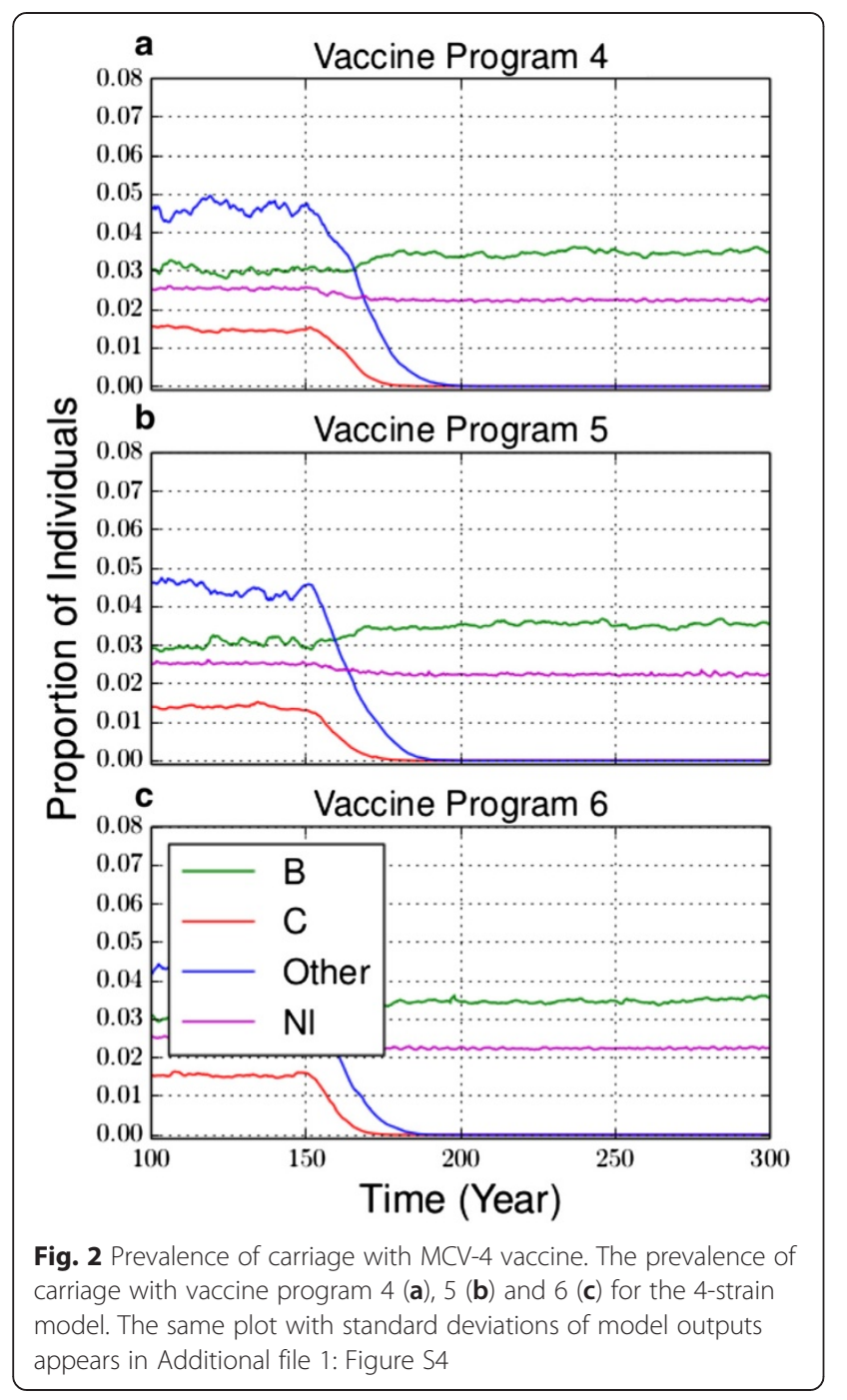

MCV-4 vaccine owing to its greater serogroup coverage than the $\mathrm{C}$ vaccine.

\section{CMen B vaccine}

According to the 4-strain model, $4 \mathrm{CMenB}$ vaccine causes the prevalence of carriage to decline at a much slower rate than the decline in serogroups $\mathrm{C}$ and "Other" when $\mathrm{C}$ and MCV-4 vaccines were introduced. This difference is on account of the lower vaccine efficacy of 4CMenB (Fig. 3a-c, see also Additional file 1: Figure S5a-c). However, as was the case for the $\mathrm{C}$ and MCV-4 vaccines, the prevalence of serogroup $B$ drops very close to zero after a sufficient amount of time.

In the 4-strain model, due to the high prevalence of serogroup $B$, the prevalence of serogroup $C$ increases significantly when the $4 \mathrm{CMenB}$ vaccine is introduced on account of strain replacement, growing to a level comparable to that exhibited by serogroup $B$ before the vaccine was introduced to the population (Fig. 3a-c, see also
Additional file 1: Figure S5a-c). More interestingly, for vaccine programs 7 and 8 , the prevalence of "Other" at first declines along with the prevalence of $\mathrm{B}$, but then rebounds, and for vaccine program 9, the prevalence of "Other" declines steeply and remains low. This suggests that changes in the prevalence of serogroup B may be pushing the prevalence of "Other" to a basin of attraction for an alternative stable state (see next subsection). We also point out that any changes in serogroup $\mathrm{C}$ prevalence (caused by vaccine-induced changes to serogroup B prevalence) would also have additional impacts on "Other" serogroup prevalence, and hence the outcomes can be more complicated than would be observed with a fewer-strain model.

The 2-strain model also predicted a slower decline under the $4 \mathrm{CMenB}$ vaccine compared to the declines under the $\mathrm{C}$ and MCV-4 vaccine (Fig. 3d-f, see also Additional file 1: Figure S5d-f). However, as was observed with the $C$ vaccine, the predicted decline in serogroup $B$ under the $4 \mathrm{CMenB}$ vaccine is significantly faster under the 2-strain model than the 4-strain model (see also Fig. 3g-i, Additional file 1: Figure S5g-i). After 30 years of immunization, the 4-strain model predicts a reduction of $65.8 \%, 61.7 \%$ and $72.4 \%$ in serogroup B for vaccine programs 7-9 respectively while the 2-strain model predicts a reduction of $93.4 \%, 93.7 \%$ and $98.5 \%$ in serogroup B for vaccine programs 7-9 respectively, over the same 30-year time period ( $\mathrm{p}=<0.0001,<0.0001$, and $<0.0001$, respectively) (Table 5 ). Both 2 -strain and 4-strain models predict that vaccinating at 12 months and 12 years of age causes the most rapid declines in prevalence (Table 5).

Unlike the 4-strain model, the 2-strain model predicts no strain replacement, which we again speculate is due to the much higher prevalence of the aggregated "Other" category, which includes Neisseria lactamica.

\section{Apparent multiple attractors}

During calibration of the 4-strain model it was noted that some parameter sets gave rise to sustained oscillations in serogroup prevalence. These were not included in the foregoing analysis. However, we present a few examples of these dynamics in Fig. 4. Although all serogroups oscillate in prevalence to some extent, the oscillations are particular pronounced for the "Other" serogroup. The $\mathrm{C}$ vaccine (programs 1-3, Fig. 4a-c) reduces the prevalence of $C$ without having a significant impact on the oscillations in the other serogroups. The MCV-4 vaccine (programs 4-6, Fig. 4d-f). However, in some simulations (such as the one depicted in Fig. 4d), strain interactions drive serogroup $B$ extinct even before any vaccines are introduced. For the $4 \mathrm{CMenB}$ vaccine, the elimination of serogroup B is accompanied by a shift in the dynamics of "Other" from sustained oscillations to 


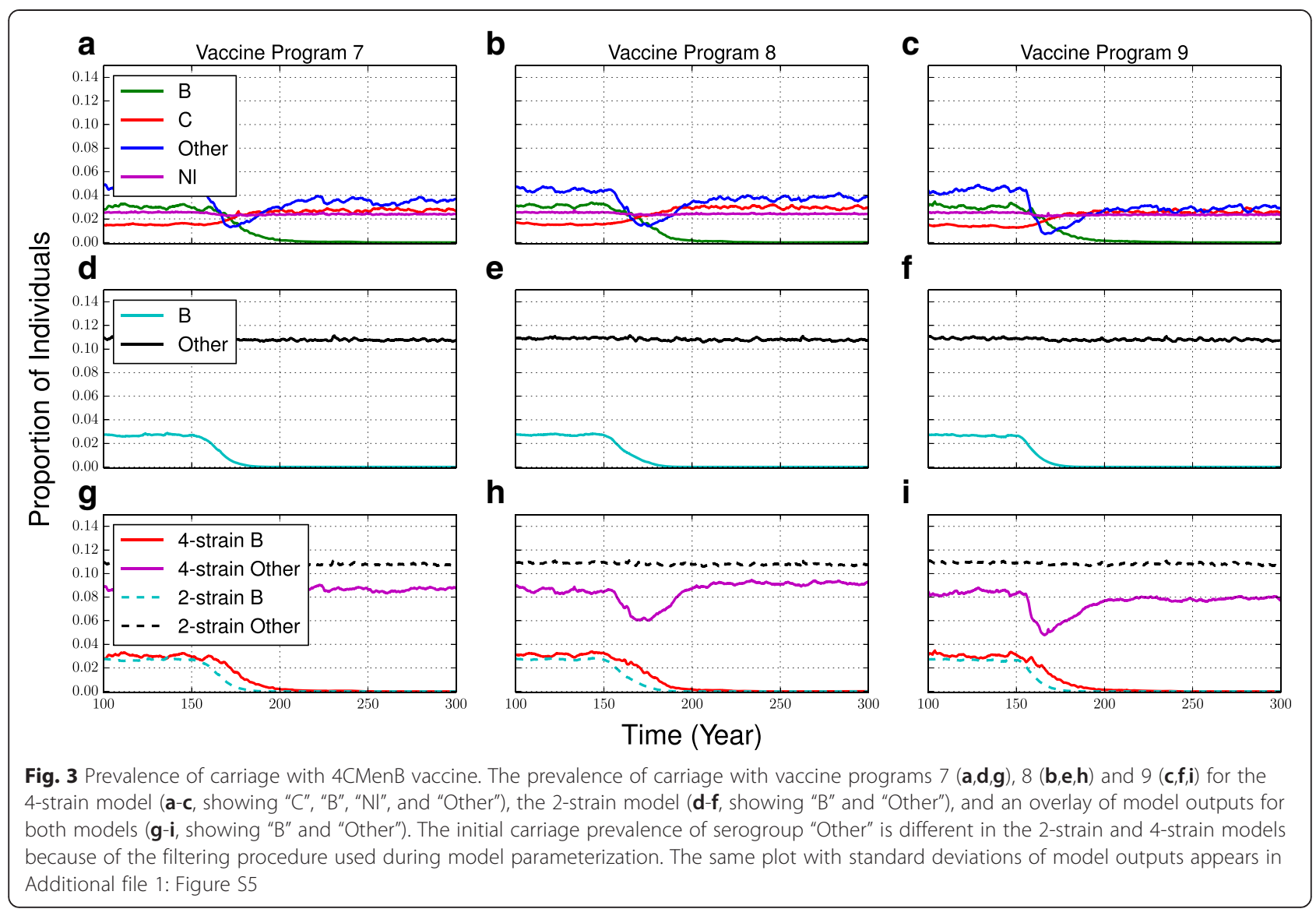

a state of high, stable (non-oscillating) prevalence (Fig. 4g-i). The prevalence of $\mathrm{C}$ also decreases significantly, probably in response to competition from a surging prevalence of "Other". The dynamics of a 4-strain model can be rich in ways that cannot be captured with a 2-strain model. We emphasize that these dynamics result from an interaction between forcing the system with the changes induced by the vaccine program, and the dynamical structure of systems with multiple attractors.

\section{Discussion}

Here we developed and compared 2-strain and 4-strain models of the impacts of several types of vaccines on Neisseria meningitidis serogroup carriage prevalence. The 2-strain and 4-strain models predicted similar overall impacts of vaccines on carriage prevalence, especially with respect to the vaccine-targeted serogroups. However, declines in vaccine-targeted serogroups were more rapid in the 2-strain model than the 4-strain model, for both the $\mathrm{C}$ and the $4 \mathrm{CMen} B$ vaccines.

Also, dynamical interactions that are present in the 4-strain model but not the 2-strain model contributed to other differences between the models. Sustained oscillations, and evidence for multiple attractors with differing basins of attraction, occurred in the 4-strain model but not the 2-strain model. In the case of the $4 \mathrm{CMenB}$ vaccine, the decline in serogroup B created an ecological niche, but because there are more than two other strains in a 4-strain model, it is not clear a priori whether serogroup C or "Other" would step in to fill the niche. In the model simulations, serogorup $\mathrm{C}$ was more successful in filling the niche, and the combined effect of changes in serogroup $\mathrm{C}$ and $\mathrm{B}$ prevalence caused the prevalence of "Other" to at first decrease, and then increase, or simply to decrease and remain low (Fig. 3d-f).

Strain replacement was more pronounced in the 4-strain model than the 2-strain model, we speculate on account of prevalence being more evenly among various serogroups in the 4-strain model, thus enhancing competitive effects. Neisseria lactamica, owing to its different natural history, did not experience the competitive effects as strongly as other $\mathrm{Nm}$ serogroups did from one another.

Oscillations have potentially important impacts on model calibration in multi-strain models. In our case, we excluded parameter sets that yielded oscillations in order to simplify the analysis. However, there are no empirical grounds for excluding such parameter sets, and oscillations in seroprevalence cannot be ruled out based on the existing and rather limited literature reporting cross- 


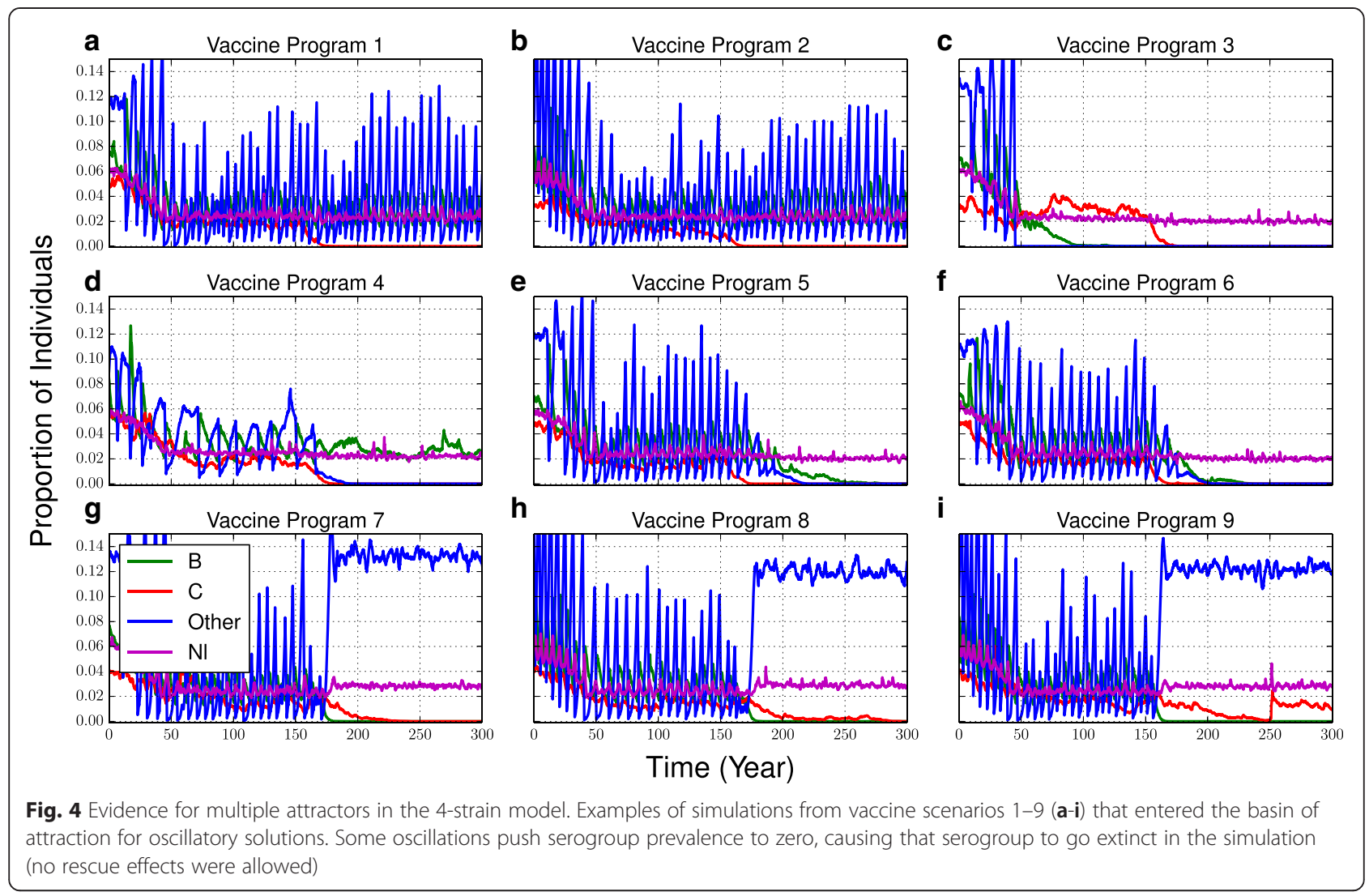

sectional results from various populations. Future models should permit oscillatory solutions that satisfy known empirical targets, especially since the qualitative and quantitative impacts of the vaccine could be very different for oscillatory solutions versus steady state solutions.

These types of dynamics appear exotic but they are not exclusive to mathematical models. There is evidence for such dynamics in other infectious disease systems, such as in pertussis before the introduction of vaccines [45]. Unfortunately, because high quality longitudinal data on $\mathrm{Nm}$ serogroup carriage prevalence do not exist, it is difficult to detect these dynamics in the case of Neisseria meningitidis. Moreover, although IMD is a notifiable disease, it is also highly stochastic, which could mask the signs of strain replacement on shorter timescales [2]. Finally, strain replacement effects can be delayed by a number of years, relative to the introduction of a vaccine program that causes them, and hence may not be immediately obvious in IMD case notification time series.

However, as more data on vaccine impacts on IMD incidence and serogroup prevalence become available over time, such new data can be used to further validate dynamic models, which can then be used to evaluate potential expansions of vaccine programs, or applications to other populations that do not currently have vaccine programs.
These post-vaccine era data can-and should-be collected so that mathematical models can better inform policy recommendations. There is also value in calibrating the same model to different local circumstances. The additional validation obtained by comparing the model under different epidemiological, vaccine, and demographic circumstances can build confidence in the models so that they may better inform policy. Further work should also explore scenarios using multiple vaccines, since many jurisdictions are currently facing the choice of how best to implement vaccination programs against multiple $\mathrm{Nm}$ serogroups.

\section{Conclusions}

The phenomena explored in these models may have implications for the predicted effectiveness of $\mathrm{Nm}$ vaccination. Caution should be exercised when determining which type of model to use to simulate Neisseria meningitis dynamics. Models with few strains are easier to analyze, but may miss important features of disease dynamics.

\section{Additional files}

Additional file 1: Supplementary Material. Additional file descriptions text (including details of how to view the file, if it is in a non-standard format). 


\section{Abbreviations}

Nm: Neisseria meningitidis; IMD: Invasive meningococcal disease; MCV4: Quadrivalent ACWY vaccine; 4CMenB: Monovalent B vaccine; NI: Neisseria lactamica.

\section{Competing interests}

CTB has received research contracts from GlaxoSmithKline Vaccines for developing models of meningococcal vaccination.

\section{Authors' contributions}

CTB conceived of the study. CTB and KP developed the model and wrote the manuscript. KP coded and simulated the model and analyzed the model outputs. Both authors read and approved the final manuscript.

\section{Acknowledgements}

The authors are grateful to support from the Natural Sciences and Engineering Research Council of Canada (NSERC) and the Canada Foundation for Innovation (CFI), and to Maria Major, Chiara Poletto, and Alberto Eugenio Tozzi for helpful comments.

\section{Received: 21 January 2015 Accepted: 6 July 2015}

Published online: 30 July 2015

\section{References}

1. $\mathrm{WHO}$ | Meningococcal Meningitis [http://www.who.int/mediacentre/ factsheets/fs141/en/]

2. Vickers, DM, Anonychuk, AM, De Wals, P, Demarteau, N, Bauch, CT Evaluation of serogroup $C$ and $A C W Y$ meningococcal vaccine programs: projected impact on disease burden according to a stochastic 2-strain dynamic model. Vaccine 2014, in press.

3. Christensen H, May M, Bowen L, Hickman M, Trotter CL. Meningococcal carriage by age: a systematic review and meta-analysis. Lancet Infect Dis. 2010;10(12):853-61.

4. PHAC Invasive Meningococcal Disease for Health Professionals [http:// www.phac-aspc.gc.ca/im/vpd-mev/meningococcal/professionalsprofessionnels-eng.php]

5. Caugant DA, Høiby EA, Magnus P, Scheel O, Hoel T, Bjune G, et al. Asymptomatic carriage of Neisseria meningitidis in a randomly sampled population. J Clin Microbiol. 1994;32(2):323-30.

6. Gauld JR, Nitz RE, Hunter DH, Rust JH, Gauld RL. Epidemiology of meningococcal meningitis at Fort Ord. Am J Epidemiol. 1965;82(1):56-72.

7. Hudson PJ, Vogt RL, Heun EM, Brondum J, Coffin RR, Plikaytis BD, et al. Evidence for school transmission of Neisseria meningitidis during a Vermont outbreak. Pediatr Infect Dis J. 1986;5(2):213-7.

8. Munford RS, de Vasconcelos ZJS, Phillips CJ, Gelli DS, Gorman GW, Risi JB, et al. Eradication of carriage of Neisseria meningitidis in families: A study in Brazil. J Infect Dis. 1974;129(6):644-9.

9. Olcen P, Kjellander J, Danielsson D, Lindquist BL. Epidemiology of Neisseria meningitidis; prevalence and symptoms from the upper respiratory tract in family members to patients with meningococcal disease. Scand J Infect Dis. 1981;13(2):105

10. Zangwill KM, Schuchat A, Riedo FX, Pinner RW, Koo DT, Reeves MW, et al. School-based clusters of meningococcal disease in the united states. JAMA. 1997;277(5):389-95.

11. Kremastinou J, Tzanakaki G, Pagalis A, Theodondou M, Weir DM, Blackwell C. Detection of igg and igm to meningococcal outer membrane proteins in relation to carriage of Neisseria meningitidis or Neisseria lactamica. FEMS Immunol Med Microbiol. 1999;24(1):73-8.

12. Troncoso G, Sanchez S, Moreda M, Criado MT, Ferreiros CM. Antigenic cross-reactivity between outer membrane proteins of Neisseria meningitidis and commensal neisseria species. FEMS Immunol Med Microbiol. 2000:27(2):103-9.

13. Gold R, Goldschneider I, Lepow ML, Draper TF, Randolph M. Carriage of Neisseria meningitidis and Neisseria lactamica in infants and children. J Infect Dis. 1978;137(2):112-21.

14. Gorringe A, Halliwell D, Matheson M, Reddin K, Finney M, Hudson M. The development of a meningococcal disease vaccine based on Neisseria lactamica outer membrane vesicles. Vaccine. 2005:23(17):2210-3.

15. Evans CM, Pratt CB, Matheson M, Vaughan TE, Findlow J, Borrow R, et al. Nasopharyngeal colonization by Neisseria lactamica and induction of protective immunity against Neisseria meningitidis. Clin Infect Dis. 2011;52(1):70-7.
16. Tzeng YL, Stephens DS. Epidemiology and pathogenesis of Neisseria meningitidis. Microbes Infect. 2000;2(6):687-700.

17. MacDonald NE, Halperin SA, Law BJ, Forrest B, Danzig LE, Granoff DM. Induction of immunologic memory by conjugated vs plain meningococcal c polysaccharide vaccine in toddlers. JAMA. 1998;280(19):1685-9.

18. MacDonald N. Immunization update 2005: Stepping forward. Can J Infect Dis Med Microbiol. 2005;16(4):219.

19. Kinlin M, Jamieson F, Brown EM, Brown S, Rawte P, Dolman S, et al. Rapid identification of herd effects with the introduction of serogroup $C$ meningococcal conjugate vaccine in ontario, canada, 2000-2006. Vaccine. 2009;27(11):1735-40.

20. PHAC Statement on conjugate meningococcal vaccine for serogroups $A, C$, Y, and W-135 [http://www.phac-aspc.gc.ca/publicat/ccdr-rmtc/07vol33/acs-03/ index-eng.php]

21. PHAC The recommended use of the multicomponent meningococcal $B$ (4CMenB) vaccine in Canada [http://www.phac-aspc.gc.ca/naci-ccni/ mening-4cmenb-exec-resum-eng.php]

22. Tan LKK, Carlone GM, Borrow R. Advances in the development of vaccines against Neisseria meningitidis. New Engl J Med. 2010;362(16):1511-20.

23. Sadarangani M, Pollard AJ. Serogroup B meningococcal vaccines-an unfinished story. Lancet Infect Dis. 2010;10(2):112-24.

24. Pillai S, Howell A, Alexander K, Bentley BE, Jiang HQ, Ambrose K, et al. Outer membrane protein (omp) based vaccine for Neisseria meningitidis serogroup b. Vaccine. 2005;23(17):2206-9.

25. Giuliani MM, Adu-Bobie J, Comanducci M, Aricò B, Savino S, Santini L, et al. A universal vaccine for serogroup B meningococcus. Proc Natl Acad Sci. 2006;103(29):10834-9.

26. Vogel U, Taha MK, Vazquez JA, Findlow J, Claus H, Stefanelli P, et al. Predicted strain coverage of a meningococcal multicomponent vaccine (4CMenB) in Europe: a qualitative and quantitative assessment. Lancet Infect Dis. 2013;13(5):416-25.

27. Hong E, Giuliani MM, Deghmane AE, Comanducci M, Brunelli B, Dull P, et al. Could the multicomponent meningococcal serogroup $B$ vaccine (4CMenB) control Neisseria meningitidis capsular group X outbreaks in Africa? Vaccine. 2013;31(7):1113-6.

28. Coen PG, Cartwright K, Stuart J. Mathematical modelling of infection and disease due to Neisseria meningitidis and Neisseria lactamica. Int J Epidemiol. 2000;29(1):180-8.

29. Caro J, Möller J, Getsios D, Coudeville L, El-Hadi W, Chevat C, et al. Invasive meningococcal disease epidemiology and control measures: a framework for evaluation. BMC Public Health. 2007;7(1):130.

30. Trotter CCL, Andrews NJ, Kaczmarski EB, Miller E, Ramsay ME. Effectiveness of meningococcal serogroup C conjugate vaccine 4 years after introduction. Lancet. 2004;364(9431):365-7.

31. Trotter CL, Gay NJ, Edmunds WJ. Dynamic models of meningococcal carriage, disease, and the impact of serogroup C conjugate vaccination. Am J Epidemiol. 2005;162(1):89-100.

32. Stollenwerk N, Maiden MCJ, Jansen VAA. Diversity in pathogenicity can cause outbreaks of meningococcal disease. Proc Natl Acad Sci U S A. 2004;101(27):10229-34.

33. Van de Velde N, Brisson M, Boily MC. Understanding differences in predictions of HPV vaccine effectiveness: A comparative model-based analysis. Vaccine. 2010;28(33):5473-84.

34. Wearing HJ, Rohani P. Ecological and immunological determinants of dengue epidemics. Proc Nat Acad Sci USA. 2006;103(31):11802-7.

35. Poletto C, Meloni S, Colizza V, Moreno Y, Vespignani A. Host mobility drives pathogen competition in spatially structured population. PLoS Comput Biol. 2013:9(8):e1003169.

36. Ballesteros S, Vergu E, Cazelles B. Influenza A gradual and epochal evolution: insight from simple models. PLoS One. 2009:4(10):e7426.

37. Nuño M, Feng Z, Martcheva M, Castillo-Chavez C. Dynamics of two-strain influenza with isolation and partial cross-immunity. SIAM J Appl Math. 2005;65(3):964-82.

38. Gumel AB. Global dynamics of a two-strain avian influenza model. Int Comput Math. 2009;86(1):85-108.

39. Statistics Canada Table 3-1 Deaths by single year of age and geography Both sexes [http://www.statcan.gc.ca/pub/84f0211x/ 2009000/t014-eng.htm]

40. Mossong J, Hens N, Jit M, Beutels P, Auranen K, Mikolajczyk R, et al. Social contacts and mixing patterns relevant to the spread of infectious diseases. PLoS Med. 2008;5(3):e74 
41. De Wals $P$, Bouckaert A. Methods of estimating the duration of bacterial carriage. Int J Epidemiol. 1985;14(4):628-43.

42. Goldschneider I, Gotschlich EC, Artenstein MS. Human immunity to meningococcus: development of natural immunity. J Exp Med. 1969;129(6):1327-48.

43. Bradet $R$, Audet $D$, Deceuninck $G$. Enquête sur la couverture vaccinale des enfants de 1 an et 2 ans au Québec en 2008. Institut national de santé publique du Québec, Direction des risques biologiques et de la santé au travail, 2009

44. An Advidory Committee Statement (ACS) National Advisory Committee on Immunization (NACl). Advice for the use of the Multicomponent Meningococcal Serogroup B (4CMenB) Vaccine. 2014.

45. Cherry J. The epidemiology of pertussis and pertussis immunization in the United Kingdom and the United State: A Comparative study. Curr Probl Pediatr. 1984;14(2):7-77.

46. White $\mathrm{CP}$, Scott J. Meningococcal serogroup C conjugate vaccination in Canada: how far have we progressed? How far do we have to go? Can J Public Health. 2010;101(1):12-4.

\section{Submit your next manuscript to BioMed Central and take full advantage of:}

- Convenient online submission

- Thorough peer review

- No space constraints or color figure charges

- Immediate publication on acceptance

- Inclusion in PubMed, CAS, Scopus and Google Scholar

- Research which is freely available for redistribution 